\title{
Building an Evidence Base on the Place of Industry-Sponsored Programs in Drug Safety Surveillance
}

\author{
Peter Arlett $^{1}$ (D) \\ Published online: 13 January 2019 \\ (c) The Author(s) 2019
}

A systems approach to pharmacovigilance ensures that planned, science-based, proportionate safety monitoring and risk minimisation are effective in supporting the safe and effective use of medicines [1]. Within pharmacovigilance systems, the reporting and analysis of individual case safety reports (ICSRs) is core to detecting new or changing safety issues (signals), and ICSRs remain a main driver for major risk minimisation actions to protect public health [2]. There is much interest in increasing the effectiveness and efficiency of reporting and analysis of ICSRs. Recent improvements have included the introduction of the International Organization for Standardization (ISO) standard for ICSRs to drive up data quality and analytical power and simplification of reporting by avoiding unnecessary duplication and increasing data access and transparency [1]. Interest also focuses on improving the methods of data analysis to detect safety signals [3], on artificial intelligence and robotics to increase efficiency and accuracy in case processing, and in making the best use of ICSRs from different sources, including from industry-sponsored programs (ISPs).

ISPs are an increasing source of ICSRs, particularly from the US. They cover a wide range of scenarios serving different purposes, with very different interactions between the company (or its contractor) and customers, who may be patients or healthcare professionals. For some drugs in some healthcare systems, ISPs may be a major part of the healthcare journey of the patient, providing high levels of contact between a company healthcare professional and a patient. Reporting obligations on the industry are based on

The views expressed in this article are the personal views of the author and may not be understood or quoted as being made on behalf of or reflecting the position of the agencies or organizations with which the author is affiliated.

Peter Arlett

Peter.Arlett@ema.europa.eu

1 Head of Pharmacovigilance and Epidemiology Department, European Medicines Agency, London, UK
International Conference on Harmonisation ( $\mathrm{ICH}$ ) principles, and require that companies record cases originating from ISPs, conduct a causality assessment, and, if the case is considered possibly causally related, to report it to the relevant regulatory systems. For some time, the industry has been arguing that ICSRs from ISPs are of low informative value and that the recording and reporting of ICSRs from them is a burden that diverts resources from more valueadding pharmacovigilance activities. Furthermore, some regulators and academics have been questioning whether overreporting by the industry, including cases very unlikely to be causally related, may obscure real signals and raise false signals [4].

The debate on the utility of ISPs in safety monitoring has been seriously undermined by a lack of good analysis and this has made evidence-based improvement of pharmacovigilance systems challenging. It is therefore very welcome that in this issue of Drug Safety, Harinstein et al. [5] report their study on ISPs. They compared the origin, seriousness, completeness and usefulness of ICSRs originating from ISPs with those not from ISPs (largely spontaneously reported ICSRs) for three biologic and three drug products. Their results show that for this sample of products, the ICSRs from the ISPs were more often serious (including more fatal cases), and they had greater completeness. The usefulness of the ICSRs based on a five-point assessment of information in the reports showed no overall difference, although the ISP ICSRs included more useful reports containing at least one serious, unlabelled reaction. These results are interesting as they suggest that ICSRs from some ISPs may be of at least equal informative value to spontaneously reported ICSRs, and that efforts to better manage and analyse them may be worthwhile. The study is limited in its size, representativeness and scope of analysis. A total of 1020 ICSRs for six different products have been analysed. The results suggest that ICSRs from different ISPs are of different completeness and usefulness. Three of the products were for oncology indications, with one each for 
haematology, cardiology and rheumatology. It is likely that the ISPs for these product types are not representative of the full diversity of ISP scenarios seen in practice. The study has very carefully assessed completeness and a measure of usefulness, however a causality assessment for each reaction in the ICSRs has not been conducted. This is important as a major value of spontaneously reported ICSRs is the consideration of the reporter that there may be a causal relationship between the product and the adverse event, with this consideration leading to the act of reporting. Depending on the case management practices of the company, this consideration of a causal relationship may be absent for some ICSRs from ISPs.

With such interest in the utility of ICSRs from ISPs in safety surveillance, we need a call to arms to the research community to provide a more comprehensive evidence base upon which we can improve pharmacovigilance. There are two broad areas for further research. First, more extensive evaluation of the profile, completeness and utility of ICSRs depending on the diverse spectrum of ISP scenarios and product types including whether some ICSRs from ISPs are duplicates of reports received spontaneously (or whether some of the reports would have been submitted spontaneously if the ISP had not existed), and, second, evaluation of the impact of these ICSRs on signal detection. The first area of research may inform whether the recording and reporting of ICSRs should differentiate between ISP type, and the second area will drive data analytic approaches to maximise the utility of the data. Noting that stratification and subgroup analysis have been demonstrated to positively impact signal detection [6], research might include analysis of the impact of inclusion and exclusion of ICSRs from ISPs on signals of disproportionate reporting in large ICSR databases. Such inclusion and exclusion could also be performed on historically detected signals and if a big enough pool of ISP ICSRs could be identified, whether the reports on their own lead to the detection of new signals. All future research, as well as any process improvements informed by that research, will be facilitated by having ISP clearly indicated in the ICSRs.

\section{Compliance with Ethical Standards}

Funding No sources of funding were used to assist in the preparation of this commentary.

Conflicts of interest Peter Arlett has no conflicts of interest that are directly relevant to the content of this commentary.

Open Access This article is distributed under the terms of the Creative Commons Attribution-NonCommercial 4.0 International License (http://creativecommons.org/licenses/by-nc/4.0/), which permits any noncommercial use, distribution, and reproduction in any medium, provided you give appropriate credit to the original author(s) and the source, provide a link to the Creative Commons license, and indicate if changes were made.

\section{References}

1. Santoro A, Genov G, Spooner A, Raine J, Arlett P. Promoting and protecting public health: how the European Union pharmacovigilance system works. Drug Saf. 2017;40:855-69.

2. Lane S, Lynn E, Shakir S. Investigation assessing the publicly available evidence supporting postmarketing withdrawals, revocations and suspensions of marketing authorisations in the EU since 2012. BMJ Open. 2018;8:e19759.

3. Wisniewski A, Bate A, Bousquet C, Brueckner A, Candore G, Juhlin $\mathrm{K}$, et al. Good signal detection practices: evidence from IMI PROTECT. Drug Saf. 2016;39:469-4903.

4. Klein K, Scholl JHG, De Bruin ML, van Puijenbroek EP, Leufkens HGM, Stolk P. When more is less: an exploratory study of the precautionary reporting bias and its impact on safety signal detection. Clin Pharmacol Ther. 2018;103:296-303.

5. Harinstein L, Kalra D, Kortepeter CM, Muñoz MA, Dal Pan GJ. An evaluation of postmarketing reports from industry-sponsored programs in drug safety surveillance. Drug Saf. 2018. https://doi. org/10.1007/s40264-018-0759-7 (Epub 19 Dec 2019).

6. Seabroke S, Candore G, Juhlin K, Quarcoo N, Wisniewski A, Arani R, et al. Performance of stratified and subgrouped disproportionality. Analyses in spontaneous databases. Drug Saf. 2016;39:355-64. 